\title{
NUEVAS PERSPECTIVAS SOBRE EDUCACIÓN
}

\author{
Dr. Marino Latorre Ariño \\ Director de la Escuela de Posgrado UMCH
}

\section{Introducción}

Si analizamos las exigencias de la universidad y de la escuela hasta hace pocos años, al estudiante universitario se le pedía adquirir una serie de conocimientos propios de la especialidad (la mayor parte de ellos teóricos) para llegar a ser un buen profesional. La práctica la aprendía pronto al llegar a la empresa y en el trabajo, pues contaba con un bagaje teórico sólido.

Al inicio del siglo XXI se exige el aprendizaje de competencias. Las competencias son capacidades-habilidades complejas que hacen referencia al manejo de conocimientos, habilidades (capacidades-destrezas) y actitudes (valores), en la realización de acciones concretas propias de una profesión y con estándares de calidad aprobados. El núcleo de una competencia es una capacidad o potencial que posee una persona para realizar determinadas acciones con estándares de calidad aceptados.

El Proyecto Tuning define las competencias como "una combinación dinámica de atributos, en relación a conocimientos, habilidades, actitudes y responsabilidades, que describen los resultados del aprendizaje de un programa educativo o lo que los estudiantes son capaces de demostrar al final del proceso educativo" (Aristimuño A. et al. 2000).

La OCDE entiende las competencias como "la capacidad de responder a demandas complejas y llevar a cabo tareas diversas de forma adecuada. Supone una combinación de habilidades prácticas, conocimientos, motivación, valores éticos y otros componentes sociales y de comportamiento que se movilizan conjuntamente para lograr una acción eficaz" (OCDE, Proyecto DeSeCo, 2005). Para Román, M. et al. (2008) el eje nuclear de las competencias 'competentes' son las capacidades, destrezas, habilidades y actitudes entendidas como herramientas mentales para aprender y seguir aprendiendo a lo largo de la vida.

Las competencias "solo son definibles y observables-evaluables en la acción"; pero una competencia no se puede reducir al saber-hacer. La competencia de un sujeto se 
reconoce en el proceso que va del saber a la acción, durante el cual se le agrega valor en forma de reacciones, decisiones y conductas exhibidas por el sujeto durante el desempeño de la tarea. Se alcanzan realizando actividades-tareas, que desarrollen habilidades mentales y compromiso personal, que permitan aprender cualquier tipo de contenidos. Partimos de que, en una programación, las unidades más elementales del proceso aprendizaje-enseñanza son "las actividades" o "tareas", que son "estrategias de aprendizaje diseñadas por el docente para que el estudiante desarrolle habilidades mentales y emocionales, y aprenda contenidos". Por ejemplo analizar un texto, sintetizar un contenido, resolver un problema, exponer un contenido ante los compañeros de clase, realizar una investigación sobre un tema concreto y exponerla ante la clase, indagar-investigar sobre temas propuestos, realizar un proyecto, etc.

La competencia profesional implica, a su vez, el desarrollo previo de las competencias académicas, que tienen relación con la competencia laboral-profesional. Pero, en realidad, hasta ahora las instituciones de educación superior, han desarrollado en el sujeto un profesional con muchos conocimientos teóricos en detrimento de la competencia laboral-profesional. Tradicionalmente las competencias laborales se dejaban para los técnicos (carreras medias, especializaciones en distintos oficios, etc.)

\section{Clases de competencias}

Presentamos una clasificación de competencias realizada por el proyecto Alfa Tuning (América Latina) que clasifica las competencias de la forma siguiente:

a) Competencias sistémicas: son competencias que permiten a la persona actuar autónomamente, como comprender el contexto en que se actúa y decide, crear y administrar planes de vida y proyectos personales y defender y afirmar los propios derechos, intereses, necesidades y límites. Entre ellas están:

* Capacidad de aprendizaje autónomo

* Capacidad para generar nuevas ideas (creatividad)

* Liderazgo

* Capacidad para el trabajo autónomo

* Iniciativa y espíritu emprendedor

* Tener voluntad y compromiso para el éxito.

b) Competencias interpersonales: son competencias que permiten interactuar en grupos heterogéneos, tales como relacionarse de forma adecuada con otros, 
cooperar y trabajar en equipo, administrar y resolver conflictos. Algunas de ellas son:

* Trabajo en equipo

* Habilidades interpersonales

* Capacidad para comunicarse con expertos de otros campos

* Capacidad para trabajar en un contexto internacional

* Compromiso ético.

c) Competencias instrumentales: capacidades cognitivas, metodológicas, tecnológicas, digitales y lingüísticas. Son las competencias que permiten dominar los instrumentos socioculturales necesarios para interactuar con el conocimiento propio de la carrera profesional, tales como el lenguaje, manejo de símbolos y números, algoritmos y conocimientos en las diversas disciplinas, así como también saber utilizar instrumentos físicos como las computadoras, máquinas, aparatos, etc.

* Conocimiento general básico

* Profundización en el conocimiento de la profesión

* Comunicación oral y escrita en el idioma propio y en extranjeros

* Habilidades básicas informáticas

* Habilidades de gestión de la información

* Resolución de problemas (pensamiento resolutivo)

* Toma de decisiones (pensamiento ejecutivo)

\section{Competencias blandas}

En la época de la globalización y en la sociedad del conocimiento se valoran mucho las llamadas "competencias blandas" (sistémicas e interpersonales). En un programa de Coaching gerencial para líderes de empresas (Becerra, T. 2016), se trataban los siguientes rubros:

Desarrollo de:

- Responsabilidad personal

- Integridad personal 
- Humildad ontológica

- Comunicación auténtica

- Coordinación impecable

- Negociación constructiva

- Inteligencia emocional

- Actitud positiva

- Red de contactos

En la Universidad de Stanford se exige a los profesores y estudiantes de educación y psicología tener un plan de vida que les permita actuar autónomamente, crear y realizar proyectos personales y defender los propios derechos, intereses y necesidades.

La Universidad Politécnica de Madrid, (2008) ha presentado el portal sobre competencias genéricas. Estas competencias genéricas para los grados son: Comunicación oral y escrita, Uso de las TIC, Respeto hacia el medio ambiente, Análisis y síntesis, Creatividad, Organización y planificación, Trabajo en equipo, Liderazgo, Idioma inglés.

Un equipo de trabajo ha realizado un exhaustivo análisis que incluye desde la demanda de esas competencias por parte de los empleadores, hasta experiencias piloto para validar la información y aportar experiencia sobre la aplicación, medición, indicadores y rúbricas de evaluación de estas competencias.

En el último documento del Word Economic Forum (2016) proponen 16 competencias para toda la vida. De ellas hay 6 competencias duras y 10 que son blandas; he aquí algún ejemplo de las blandas: Trabajo en equipo, tolerancia, curiosidad, constancia, compromiso, adaptabilidad, capacidad de liderazgo, respeto a la cultura social, empatía, respeto, etc.

Hoy en día, en el mundo globalizado en que vivimos y en la sociedad del conocimiento, cada día se valoran más las competencias blandas para hacer frente a las demandas de los trabajos profesionales. En la sociedad industrial era necesario el saber $y$, si era posible, saberlo todo sobre una materia, pero hoy en día la mayor parte del conocimiento y la tecnología está en las máquinas y se tiene acceso con un clic; por eso, el profesional tiene que privilegiar la gestión de la información y de la tecnología y su capacidad para actuar de forma creativa, organizar y planificar, tomar decisiones, la capacidad de crítica y autocrítica, el aprendizaje autónomo, el compromiso personal, el trabajo en equipo, las habilidades interpersonales, etc. 
Todas estas son las llamadas competencias blandas. El aprendizaje dialógico permite desarrollarlas. De ahí su importancia.

\section{El aprendizaje dialógico y la personalización y socialización}

La personalización se consigue viviendo con otras personas, pues el ser humano nace, vive y se desarrolla en comunidad y necesita de los otros para la supervivencia y para llegar a ser persona. "El hombre es un ser social por necesidad y porque lo requiere así la naturaleza de su mente" (Henz, H. 1968). Los conocimientos, costumbres, hábitos, valores, creencias, patrones de conducta, etc. son trasmitidos de una generación a otra. Las comunidades modernas convierten al individuo cada vez más dependientes unos de otros. No podemos escapar de este entramado social.

La socialización no es algo genético, no es innato, no es un rasgo biológico de la personalidad. La socialización es un aprendizaje. Se nace radicalmente referido a los demás, pero se aprende a respetarles, a tolerarles, a comprenderles y a ayudarles. Uno de los fines de la educación es el proceso de socialización. Aprender a vivir juntos y convivir con los demás. Aristóteles decía que el hombre es un "animal político" -habitante de la polis (ciudad), ámbito social por excelencia--. A través del proceso de socialización se aprenden las normas que rigen en una sociedad y se asimilan los patrones sociales y culturales de un pueblo.

La preocupación porque la enseñanza tenga un verdadero acento socializador ha dado lugar a la aparición de instituciones escolares y a la propagación de contenidos y actividades realizadas en dinámica grupal. Se han inventado gran cantidad de técnicas en trabajo grupal como ABP, APP, aprendizaje por indagación, aprendizaje dialógico, estudio de casos, estudio de dilemas morales, etc. Esta metodología permite el desarrollo de habilidades blandas, así como el aprendizaje de contenidos de las diversas áreas. Vamos a detenernos en el aprendizaje dialógico.

\section{El aprendizaje dialógico}

El aprendizaje dialógico se basa en la comunicación entre personas; se sabe que las personas aprendemos a partir de las interacciones con otras personas. A través del habla y comunicación con otras personas el sujeto da sentido a su realidad. Como afirma Vygostsky (1996), "construimos el conocimiento primeramente desde un plano inter-subjetivo, es decir, desde lo social, a través de la interacción con otras personas y progresivamente lo interiorizamos como un conocimiento propio (intra-subjetivo)". El proceso de internalización es el paso de la inter-subjetividad a la intra-subjetividad. La internalización es la representación interna -personal-- de un fenómeno externo 
-social--. Equivale al proceso de "adaptación-equilibrio" de Piaget como un equilibrio de asimilación-resolución del conflicto cognitivo-adaptación.

Puesto que la inteligencia es un potencial cognitivo y emocional flexible y moldeable, que se desarrolla en el sujeto en función de las oportunidades que tiene de aprender en los contextos sociales y culturales en que vive, la inteligencia cultural es el conjunto de conocimientos y capacidades que posee una persona que vive en contextos determinados, (lenguaje, conocimientos teóricos y prácticos, tradiciones, creencias, costumbres, hábitos, etc.).

Los test tradicionales de Binet y Simon miden el C. I. Estos test tradicionales miden lo que un estudiante puede hacer solo, pero no lo que puede hacer con ayuda de otros, colaborando con ellos. Hoy en el ámbito laboral es más importante lo que puede hacer una persona con otros que lo que puede hacer solo. El poner a disposición de la comunidad nuestros propias aptitudes, actitudes y valores para resolver ciertos problemas es llamada la "inteligencia distribuida" (Pea, 1993; Wertsch, 1998).

La inteligencia cultural tiene tres manifestaciones: inteligencia académica, inteligencia práctica e inteligencia comunicativa.

a) Inteligencia académica (la que se desarrolla en contextos académicos). Es la que medía hace unos años el C. I., a través de los test.

b) Inteligencia práctica: la inteligencia práctica (la que se desarrolla en contextos cotidianos y en las relaciones de la sociedad informal) (Wagner y Sternberg ( 1986). Es la inteligencia que se desarrolla haciendo cosas; es el aprendizaje manipulativo; el trabajo manipulativo -el pensar con las manos-- desarrolla la inteligencia igual que el trabajo especulativo, aunque de forma diferente.

La inteligencia académica no es más importante que la inteligencia práctica. Todo depende de los contextos en que se usen las inteligencias. Por ejemplo: Rosa es doctora en Educación y especialista en Epistemología e investigación; Susana es mecánica de taller de carros. Si mi carro tiene un desperfecto mecánico, pensaré en Susana antes que en Rosa.

c) Inteligencia comunicativa. Todas las personas tienen inteligencia comunicativa; incluye la habilidad para utilizar el lenguaje y otras formas de comunicación para explorar el mundo y resolver problemas. No olvidemos que el ser humano está dotado de inteligencia para poder sobrevivir y para conocer el mundo en que vive. 
El diálogo debe estar basado en una relación de igualdad y no de poder, lo que significa que todas las personas tienen conocimiento que aportar, reconociendo así, la inteligencia cultural en todos. Mediante el diálogo transformamos las relaciones, nuestro propio conocimiento y el entorno. De este modo "el aprendizaje dialógico se produce en interacciones que aumentan el aprendizaje instrumental, favorecen la creación de sentido personal y social, y está guiado por principios solidarios y en los que la igualdad y la diferencia son valores compatibles y mutuamente enriquecedores" (Aubert, A.; Flecha, A.; García, C.; Flecha, R.; Racionero, S., 2008).

\section{Vigotsky y la importancia de la inteligencia comunicativa}

La teoría de Vigotsky es que el sujeto aprende en contextos culturales concretos y usa como herramientas para aprender el "pensamiento y el lenguaje" del contexto en que se encuentra (procesos psicológicos superiores).

El desarrollo de la inteligencia tiene por fin "transformar el contexto" y no "adaptarse a él"... En educación no se trata de bajar los niveles de exigencia o reducir la cantidad de contenidos o competencias para los estudiantes de contextos desfavorecidos (adaptaciones curriculares), sino de exigirles igual que a todos; la única manera de transformar la realidad es transformándo a los estudiantes, es decir, desarrollando su inteligencia de forma integral (lo cognitivo y lo emocional).

El arte del profesor es saberse mover en la zona de desarrollo próximo, (ZDPróx.) poniendo al estudiante en situaciones de mayor dificultad a medida que avanza el curso y el nivel de estudios. El máximo aprendizaje se alcanza por interacciones heterogéneas (Vigotsky: ZDPróx y ZDPot..., Bruner, (2000) etc. En el trabajo interactivo y dialógico se desarrollan habilidades de todo tipo: académicas, teóricas, prácticas y comunicativas.

Las habilidades comunicativas desarrollan sobremanera la inteligencia:

- Razonamiento verbal,

- Explicar conceptos,

- Organizar procesos lógicos y coherentes

- Argumentar

- Interpretar, analizar, inferir, etc.

- Entonación, ritmo, estructuración del pensamiento y del discurso. 
La comunicación dialógica tiene como objetivo que el compañero aprenda lo que no sabe a través de la explicación de otro que sabe más que él; es el aprendizaje por tutoría'; en el proceso aprenden todos: los que no saben y los que saben... El que sabe y explica, aprende a explicar, a estructurar su pensamiento, a relacionarse con el otro, a desarrollar valores como paciencia, solidaridad, etc. La práctica se desarrolla a través de preguntas, no de respuestas. La esencia de la relación tutoral es dar el mayor control posible al aprendiz sobre lo que quiere aprender y organizar el diálogo sobre el descubrimiento que él debe hacer, ayudado por el tutor, para no sólo entender lo que escogió, sino cómo se estructura lo que ahora entiende. La tutoría lleva a razonar y a descubrir, no a encontrar pronto la respuesta que el maestro espera o proporciona.

Las preguntas que se hacen en la tutoría no son para inducir "respuestas correctas" sino para profundizar la comprensión del tema tanto por parte del aprendiz como del mismo tutor. En este contexto lo inesperado es lo más esperado. El tutor, independientemente de las veces que haya dado tutoría sobre un tema, espera siempre sorpresas en la manera particular como su aprendiz aborda y asimila el tema.

Hay muchas posibilidades de desarrollo para un adolescente, pero verbalizar ${ }^{2}$ es una de las más importantes. El lenguaje ofrece múltiples y diversas oportunidades de desarrollo. Los aprendices en la tutoría, por ejemplo, desarrollan un amplio vocabulario con el que expresan empatía y ayudan a sus compañeros. El simple hecho de permanecer uno frente al otro durante un buen tiempo hace necesariamente que jóvenes o adultos generen diálogos de gran complejidad y muy variados. Los jóvenes tutores ensayan distintas preguntas para lograr descubrir lo que impide a sus aprendices avanzar en la comprensión de un problema o de un texto.

¿Cómo se percibe la acción que se realiza? A través de los sentidos. ¿Cómo se expresa la acción que se realiza? A través de la verbalización externa, razonando en voz alta, describiendo la acción, etc.

Esta es la mejor manera de aprender pues posibilita la realización de sinapsis entre las neuronas y el desarrollo de la inteligencia. La comprensión individual siempre se consigue como "resultado de haber logrado la intersubjetividad y el pensamiento colectivo" (Rogoff, 1993, p. 227).

1 La palabra tutoría o tutor es empleada aquí como sinónimo de profesor-mediador; se refiere a la persona (sea docente o estudiante, cualificado en un tema o materia) que enseña a otros que saben menos que él. Está basado en el método mutuo lancasteriano del siglo XIX. Consiste en que los estudiantes, que saben más de un tema, puedan explicarlo a los que saben menos; estos alumnos hacen de docentes temporales en el aula. Después estos docentes temporales se convierten en alumnos de otro compañero que sabe más que ellos sobre otro tema concreto.

2 Verbalizar: Según la teoría Sociocrítica de aprendizaje (Vygostky y seguidores) la adquisición y dominio de los instrumentos culturales (lenguaje oral o escrito, símbolos matemáticos, musicales, etc.) posibilita la apropiación de los aprendizajes. El estudiante adquiere la cultura del entorno a través de la internalización. La internalización es la reconstrución interna de una acción externa. 
La universidad de Harward, por ejemplo, pone todo su empeño en que en sus aulas haya mucha heterogeneidad (cultural, lingüística, social, religiosa, económica, etc.); han experimentado que con grupos heterogéneos se aprende más y mejor. La heterogeneidad en las aulas es un factor que conduce a la excelencia académica. El MIT (20 I 6) hay estudiantes de I 52 países; es una pequeña torre de Babel con unos I I,300 estudiantes de toda raza, lengua, pueblo y nación. Su educación está basada en la colaboración, no en la competición.

\section{Lo que sí funciona en educación}

Ordinariamente los profesores tenemos casi como un paradigma que "si el profesor explica algo correctamente a los estudiantes en el aula, éstos lo aprenden". Es una apreciación incorrecta porque "solo se aprende lo que se quiere y se puede aprender", pues "el aprendizaje no se produce cuando alguien -el profesor-quiere enseñar, sino cuando alguien -el estudiante-- quiere y puede aprender". Elmore, R. (2016) hablando de su experiencia afirma que en los salones de clase que observó en escuelas norteamericanas encontró un patrón estable de un 85-90 \% de habla de adultos y 10 - I 5 \% de habla de los estudiantes. Los adultos, en general, hablaban en largos y ampulosos períodos, frecuentemente con frases o párrafos incompletos. Los estudiantes, por lo general, hablaban brevemente, frases de menos de doce palabras; también con fases incompletas.

En sus observaciones de salones de clase de escuelas norteamericanas registraba regularmente el lenguaje corporal, las miradas y expresiones verbales que revelan interés de los estudiantes por aprender. De esas observaciones surgió lo que he dado en llamar "la regla de tercios". Aún en las mejores circunstancias, que no eran las más comunes:

- Un tercio de la clase parecía no tener interés alguno por lo que se explicaba en la clase (vista perdida, cuerpo suelto, postura relajada, etc.).

- Otro tercio parecía formalmente interesado (mostraban atención, pero no en sintonía con lo que hacía y decía el maestro; oían y poco más, sin molestar...) y

- El otro tercio estaba realmente interesado (mirándose y viendo al maestro, preguntando, asintiendo, etc.) (Elmore, 20।6).

Las manifestaciones de interés en algunos estudiantes variaban, pero la proporción de tercios se mantenía constante. Aunado a este comportamiento se daba otro más desolador. Cuando se preguntaba a los estudiantes qué estaban estudiando, --mostraran interés o no en el estudio--, sus respuestas eran estas: decían que lo que 
enseñaba el maestro (no decían lo que estaban aprendiendo) o decían, con sencillez, que no sabían lo que estaban aprendiendo.

Barrientos, R. (2016) muestra lo que la investigación dice que sí funciona, para mejorar aprendizajes. El investigador neozelandés Hattie, J. (20I2) le ha dedicado más de treinta años de su vida a tratar de desentrañar los misterios del aprendizaje. Hattie es investigador de la universidad de Auckland (Nueva Zelanda) y realizó durante I 5 años más de 800 metanálisis junto con una revisión de 50 mil artículos de investigación relacionados con el logro en los aprendizajes, utilizando una data que involucraba a más de 250 millones de estudiantes. Su esfuerzo buscó desvelar los elementos de la práctica docente efectiva, aquella que logra "hacer visible la enseñanza para los estudiantes y el aprendizaje para los profesores" (p. 25).

Hattie realizó un metanálisis --un ejercicio estadístico-- de la data de cientos de investigaciones sobre un determinado tema: tareas escolares, enfoques de lectura o matemáticas, etc. El gran aporte de los metanálisis es que, a través de él, se realiza una síntesis estadística de mucha información lo que ofrece una mayor solidez para realizar generalizaciones. Los descubrimientos realizados por los metanálisis nos ofrecen indicios sobre dónde buscar la buena práctica pedagógica (Glass, MacGaw, \& Smith, 1984). El tamaño del efecto muestra el grado de incidencia o contribución de una determinada variable sobre el logro de aprendizajes. A partir de $0.4 / /$ se puede considerar como efectos positivos de impacto importante sobre aprendizaje. Los metanálisis se usan en el mundo de la salud y otros ámbitos desde hace un buen tiempo, y es una herramienta útil para elaborar políticas y tomar decisiones.

\section{8. ¿Cuáles son las estrategias educativas con mayor efecto en los aprendizajes?}

Las cinco prácticas del docente que, según los cientos de metanálisis mostraron, generan mayores logros de aprendizaje para todos los estudiantes:

$\checkmark$ La enseñanza mutua-recíproca,

$\checkmark$ La retroalimentación del profesor al estudiante,

$\checkmark$ La auto-verbalización y el auto-cuestionamiento,

$\checkmark$ Las estrategias metacognitivas,

$\checkmark$ La resolución de problemas (J. Hattie, 2008).

En la tabla siguiente se presenta cada una de ellas con su respectivo tamaño del efecto. 
Tabla I. Estrategias de enseñanza con mayor efecto en los logros de aprendizaje

\begin{tabular}{|l|c|}
\multicolumn{1}{|c|}{ Estrategia } & Tamaño del efecto \\
\hline Enseñanza mutua-recíproca & $0.74 / 1$ \\
\hline Retroalimentación-feedback, & $0.74 / 1$ \\
\hline Auto-verbalizar y auto-cuestionarse & $0.64 / 1$ \\
\hline Las estrategias metacognitivas & $0.69 / 1$ \\
\hline Resolución de problemas & $0.61 / 1$ \\
\hline
\end{tabular}

Fuente: Elaboración a partir de Hattie, 2008.

Explicamos brevemente cada una de ellas.

\section{$\checkmark$ La enseñanza mutua-recíproca}

La enseñanza recíproca tiene un tamaño del efecto alto, 0.74/I . En esta estrategia los docentes permiten que los estudiantes aprendan entre ellos y hagan uso del autoaprendizaje. Éstos asumen un rol de tutor para sus compañeros. Para el análisis de esta variable se realizó una síntesis de 38 estudios sobre el tema.

Los países con mayor calidad de enseñanza han focalizado sus esfuerzos en capacitar a los docentes en el dominio efectivo del aprendizaje colaborativo a través de la enseñanza recíproca.

\section{$\checkmark$ La retroalimentación o feedback}

La segunda práctica con mayores efectos en logros de aprendizaje es la retroalimentación o feedback, con un $0.73 /$ l de tamaño del efecto. Consiste en ofrecer respuestas o retroalimentación al trabajo de los estudiantes. Es decir se realiza la evaluación para el aprendizaje y como una forma de aumentar el aprendizaje (Sharratt \& Fullan, 2012). Es decir, que "el estudiante no aprende para ser evaluado, sino que es evaluado para aprender" (Gómez Parra, S., 20 I0, p. 5).

Marzano y Heflebower (2010) realizaron una investigación para medir el tamaño del efecto del feedback tomando en cuenta en número de veces que se realizaba y descubrieron algunos aspectos interesante. En la tabla I se puede observar que a mayor número de retroalimentaciones realizadas por el docente en un periodo de I 5 semanas mayor era el tamaño del efecto para logros de aprendizaje. 
Tabla I. Tamaño del efecto del feedback a lo largo de I 5 semanas.

\begin{tabular}{|c|c|}
\hline $\begin{array}{c}\text { Número de evaluaciones } \\
\text { retroalimentaciones }\end{array}$ & Tamaño del efecto \\
\hline 0 & 0 \\
\hline 1 & $0.34 / 1$ \\
\hline 5 & $0.53 / 1$ \\
\hline 10 & $0.6 / 1$ \\
\hline 15 & $0.66 / 1$ \\
\hline 20 & $0.71 / 1$ \\
\hline 25 & $0.78 / 1$ \\
\hline 30 & $0.82 / 1$ \\
\hline
\end{tabular}

Fuente: Marzano y Hefleboweer, 2010.

Se podría deducir de esta información que realizar muchas evaluaciones a los estudiantes es positivo. Sin embargo, cuando se realizó una síntesis de diversos estudios, ésta mostró que no siempre realizar evaluaciones continuas es productivo.

Muchas veces puede tener efectos contrarios a lo esperado. Por esta razón es prudente ser cautos con el uso de la evidencia. Lo que podemos concluir es que no cualquier evaluación produce la mejora del aprendizaje; además realizar evaluaciones sumativas semanales no siempre es bueno para que los estudiantes aprendan. La mejor retro-alimentación es la descriptiva. Ésta requiere mucho entrenamiento y trabajo en equipo para llegar a dominarla.

\section{$\checkmark$ Las estrategias metacognitivas}

Las otras dos estrategias más potentes son el enseñar a los estudiantes a auto-verbalizar. Consiste en ofrecer a los alumnos espacios para que verbalicen lo que tienen que realizar, cómo lo realizarán y cómo lo han realizado. Esta estrategia tenía un 0.64/I de tamaño del efecto y se realizaron tres metanálisis de II3 estudios. La estrategia se puede enmarcar dentro de las estrategias para favorecer la metacognición. Las estrategias metacognitivas tienen un tamaño del efecto de $0.69 / 1$.

\section{$\checkmark$ La resolución de problemas}

Por último, la resolución de problemas es otra de las estrategias con un alto impacto en la mejora de logros de aprendizaje con un 0.6I/I de tamaño del efecto. Para la misma se realizaron 6 metanálisis de 221 estudios. 


\section{ANEXO I}

\section{Factores extra e intraescolares que impactan más en los logros de aprendizaje}

El análisis realizado por Hattie (20/2) tuvo en cuenta diversas dimensiones de la vida escolar: factores relacionados con los estudiantes, factores sobre el hogar, características de las escuelas, características del docente, enfoques curriculares y por último, estrategias del trabajo docente. En la tabla siguiente se presenta una síntesis de aquellas variables que tienen mayor impacto en la mejora de aprendizajes.

Tabla. Efecto de los principales factores que influyen en los aprendizajes según Hattie.

\begin{tabular}{|c|c|c|c|c|c|}
\hline Estudiante & Hogar & Escuela & Docente & Currículum & Trabajo en clase \\
\hline \multirow[t]{2}{*}{$\begin{array}{l}\text { Programas } \\
\text { pre- } \\
\text { escolares } \\
0,45\end{array}$} & \multirow{5}{*}{$\begin{array}{l}\text { Involucración } \\
\text { de la familia en } \\
\text { el aprendizaje } \\
\text { del estudiante } \\
0,51\end{array}$} & $\begin{array}{l}\text { Gestión del } \\
\text { salón de } \\
\text { clase } \\
0,52\end{array}$ & $\begin{array}{l}\text { Microen- } \\
\text { señanza } \\
0,83\end{array}$ & Comunicación & $\begin{array}{l}\text { Retroalimentación } \\
0,73\end{array}$ \\
\hline & & $\begin{array}{l}\text { Cohesión } \\
\text { escolar } \\
0,53\end{array}$ & $\begin{array}{l}\text { Calidad de la } \\
\text { enseñanza } \\
0,44\end{array}$ & \multirow{2}{*}{ Matemática } & \multirow{2}{*}{$\begin{array}{l}\text { Tutoría } \\
\text { entre pares } \\
0,55\end{array}$} \\
\hline \multirow[t]{3}{*}{$\begin{array}{l}\text { Intervención } \\
\text { temprana } \\
0,47\end{array}$} & & \multirow{2}{*}{$\begin{array}{l}\text { Orden y } \\
\text { disciplina en } \\
\text { clase } 0,80\end{array}$} & $\begin{array}{c}\text { Re la ción } \\
\text { estudiante- } \\
\text { profesor. } \\
\quad 0,72\end{array}$ & & \\
\hline & & & $\begin{array}{c}\text { Desarrollo } \\
\text { docente } \\
0,62\end{array}$ & \multirow{2}{*}{ Ciencias } & \multirow{2}{*}{$\begin{array}{l}\text { Ciencias } \\
\text { Estrategias meta- } \\
\text { cognitivas } \\
0,68\end{array}$} \\
\hline & & $\begin{array}{l}\text { Influencia de } \\
\text { pares } \\
0,53\end{array}$ & $\begin{array}{l}\text { Expectativas } \\
\text { del docente } \\
0,43\end{array}$ & & \\
\hline
\end{tabular}




\section{Referencias}

Aristimuño, A., González, M., y Luján, C. (2000). Organización y docencia universitaria: un diálogo posible. El reciente proceso de desarrollo institucional de la Universidad Católica del Uruguay. Primer Congreso Internacional "Docencia Universitaria e Innovación". Universidad Autónoma de Barcelona, Universidad de Barcelona, Universidad Politécnica de Catalunya.

Aubert, A.; Flecha, A.; García, C.; Flecha, R.; Racionero, S. (2008). Aprendizaje Dialógico en la Sociedad de la información. Barcelona: Hipatia.

Barrientos, R. (2016). Lo que sí funciona en educación, en Revista Educa UMCH, $\mathrm{n}^{\circ}$ 7, agosto 2016, p. 87.

Becerra, T. (2016). Diplomado de "Conscious Business Coaching aplicado al Liderazgo consciente". Lima.

Bruner, J. (2000). La educación, puerta de la cultura. Madrid: Visor.

Cooper, H. (I 989). Homework versus no-treatment: Longman.

Elmore, R. (2016). Reflexiones sobre la contribución de la tutoría al futuro del aprendi-zaje, en Revista Educa UMCH, n 7, pp. 97-I I0.

Glass, G. V., MacGaw, B., \& Smith, M. L. (1984). Meta-analysis in social research: Sage Beverly Hills, CA.

Gómez, S. (2010). Situaciones de aprendizaje y evaluación. Revista Padres y Maestros, ISSN 0210-4679, n 329, 2010, págs. 5-9.

Hattie, J. (2008). Visible learning: A synthesis of over 800 meta-analyses relating to achievement: Routledge.

Hattie, J. (2012). Visible Learning for Teachers: maximizing impact on learning: Routledge.

Henz, H. (1968). Tratado de pedagogía sistemática. Barcelona: Herder.

Latorre, M. (20|4). Diseño curricular por capacidades y competencias en educación superior. Universidad Marcelino Champagnat: Lima. 
Marzano, R. J., \& Heflebower, T. (20।0). Formative assessment \& standards-based grading: Solution Tree.

MIT (20 I6). Entrevista a Rafael Reif, presidente del MIT. El País, 25/I0/20 I6, p. 22.

OCDE, (2005). El Proyecto DeSeCo (Definición y Selección de Competencias Clave).

Pea, R. (1993). Prácticas de inteligencia distribuida y diseños para la educación, en Cogniciones distribuidas. Buenos Aires: Colección Agenda Educativa. Amorrortu.

Proyecto Tuning, (2002). Deusto, Bilbao, España. http://www.relint.deusto.es/ TUNINGProject/general-conclusions.asp

Rogoff, B. (1993). Aprendices del pensamiento. El desarrollo cognitivo en el contexto social. Barcelona. Paidós.

Román, M. y Díez, E. (2008). Diseño curricular de aula: Modelo T. Puerta de entrada en la Sociedad del conocimiento. Santiago de Chile, Chile: Conocimiento.

Sharratt, L. y Fullan, M. (20 I2). Putting FACES on the Data: What Great Leaders Do!. Corwin Press.

Universidad Politécnica de Madrid (2008). Recuperado de: http://innovacioneducativa. upm.es/sites/default/files/apoyo_profesorado/Recomendaciones\%20 para\%20la\%20implantaci\%C3\%B3n\%20del\%20modelo\%20en\%20 las\%20titulaciones\%20de\%20la\%20UPM.pdf

Vygotsky, L. (1996). El desarrollo de los procesos psicológicos superiores. Barcelona: Crítica.

Wertsch, K. J. ( 1998). Mind as action. Nueva Yoek: Oxford.

Word Economic Forum (2016). New Vision for Education: Fostering Social and Emotional Learning through Technology. 